\title{
Nonlinear PI control of uncertain systems: an alternative to parameter adaptation
}

\author{
Romeo Ortega $^{\mathrm{a}, *}$, Alessandro Astolfi ${ }^{\mathrm{b}, \mathrm{c}}$, Nikita E. Barabanov ${ }^{\mathrm{d}}$ \\ ${ }^{a}$ Laboratoire des Signaux et Systémes, CNRS-Supelec, Plateau du Moulon, 91192 Gif-sur-Yvette, France \\ ${ }^{\mathrm{b}}$ Dipartimento di Elettronica e Informazione, Politecnico di Milano, Milan, Italy \\ ${ }^{\mathrm{c}}$ Electrical Engineering Department, Imperial College, London, SW7 $2 B T$ UK \\ ${ }^{\mathrm{d}}$ Department of Software Engineering, Electrotechnical University, St Petersburg, Russia
}

Received 30 January 2001; received in revised form 15 May 2002; accepted 4 June 2002

\begin{abstract}
A novel approach to stabilization and trajectory tracking for nonlinear systems with unknown parameters and uncertain disturbances is developed. We take a drastic departure from the classical adaptive control approach consisting of a parameterized feedback law and an identifier, which tries to minimize a tracking (or prediction) error. Instead, we propose a simple nonlinear PI structure that generates a stable error equation with a perturbation function that exhibits at least one root. Trajectories are forced to converge to this root by suitably adjusting the nonlinear PI gains. We consider the two basic problems of: (i) matched uncertainties, when the uncertain terms are in the image of the input matrix, and (ii) unknown control directions, when the control signal is multiplied by a gain of unknown sign. We show that, without knowing the system parameters, and with only basic information on the uncertainties we can achieve global asymptotic stability and global tracking, without injecting high gains into the loop. Interestingly, we prove that we can take as our nonlinear PI structure an activation function reminiscent of that used in neural networks. Although most of the results are derived assuming full state measurement, we also present an observer-based solution for a chain of integrators with unknown control direction. The procedure is shown to provide simple solutions to the classical problems of neural network function approximation, as well as eccentricity control and friction compensation of mechanical systems.
\end{abstract}

(c) 2002 Elsevier Science B.V. All rights reserved.

\section{Introduction}

It is well known that (linear) PI controllers, if suitably tuned, provide satisfactory solutions to many practical applications without requiring a detailed description of the system dynamics. In the presence of strong nonlinear effects, however, their performance is below par, and it is necessary to "re-tune" the controller appealing to gain scheduling or adaptive procedures. The design of these tuning procedures is complicated when only

\footnotetext{
* Corresponding author. Tel.: +33-1-6985-1748; fax: +33-1-6941-3060.

E-mail addresses: romeo.ortega@1ss.supelec.fr (R. Ortega), a.astolfi@ic.ac.uk (A. Astolfi), nikita@freya.etu.ru (N.E. Barabanov).
} 
a very coarse description of the uncertain nonlinearities is available-some prototypical examples being the presence of friction and eccentricity in mechanical systems, and the lack of knowledge about the reaction functions in chemical and biological processes. In the adaptive control approach it is assumed that we can fit a model-e.g., an experimental data-fitting curve or a neural network - to the uncertain function. Then, a parameter identifier, which tries to minimize a tracking (or prediction) error, is implemented. To handle the curse of dimensionality, the parameterization has to be nonlinear, see e.g., [10,24]. Functional approximation with nonlinearly parameterized models is, of course, a fundamentally difficult problem to which an enormous amount of research has been devoted. ${ }^{1}$ Several interesting applications have been reported in the control community, with a central contribution being the observation that convexity can be exploited to ensure a "good gradient descent" in some regions of the state space [6], see also [7,1]. Unfortunately, to the best of our knowledge, to cope with the remaining regions all provably stable schemes rely on one way or another on relay actions or min-max calculations - hence, the stabilization mechanism is of high gain nature, see, e.g. $[22,15]$ and the references therein. Two additional drawbacks of adaptive methods are that they usually lead to highly complex designs, and significant prior knowledge is required for a successful operation. (See also [25] for an alternative treatment of this class of problems.)

For some particular system structures it is possible - but not necessarily very practical - to crank up the gain and try to "dominate" the uncertainties. High-gain designs appear in the nonlinear control literature in many forms, including relay actions, min-max calculations, and recursive "domination" via high-order nonlinear terms. ${ }^{2}$ The deleterious effects of injecting high gain in the control loop, even though well known by practitioners, are unfortunately not fully appreciated by the theoretical control community.

In this paper, pursuing the line of research started in [3], we adopt a radically different perspective to the problem, abandoning its parametric formulation. Namely, instead of trying to fit a parameterized function to the uncertainty (or dominate it), we aim at the-less ambitious but equally effective-objective of generating a stable error equation with a perturbation term that can be driven to zero. More precisely, choosing the functions that define our nonlinear PI controller, we "shape" the perturbation function so as to exhibit at least one root, towards which we force the trajectories to converge. We show in the paper that these tasks can be achieved for a broad class of control problems with very little prior knowledge on the uncertainties. To illustrate this point we consider the two basic problems of: (i) matched uncertainties, when the uncertain terms are added to the input channels; and (ii) unknown control directions, when the control signal is multiplied by a gain of unknown sign. In the former case we show that, with only a coarse knowledge on the uncertainties we can achieve global asymptotic stability and global tracking, without injecting high gains into the loop. For the unknown control directions problem, our design yields an alternative to the well-known Nussbaum gain stabilizers [16] that - in contrast with the latter and due to the presence of the proportional term in the PI-is shown to be robustly stable vis-à-vis (fast) unmodeled dynamics and measurement noise. (See also [12].) Although most of the results are derived assuming full state measurement, we also present an observer-based solution for a chain of integrators with unknown control direction.

Even though some of the examples we consider can be stabilized applying existing control-plus-estimation or high-gain designs, it is our contention that the schemes proposed in this paper will, in general, exhibit a better performance and be easier to commission. These claims are substantiated by the following facts:

- In contrast with adaptive control, where the search takes place in a high-dimensional (and/or topologically complex) parameter space, the "root search" described here happens in a one-dimensional space.

\footnotetext{
${ }^{1}$ Actually, as clearly illustrated in the tutorial paper [19], even for linear systems with linearly parameterized models, many fundamental issues remain open.

${ }^{2}$ The latter includes, of course, the widely popular backstepping method [14] that has proven itself very versatile. See also [13].
} 
- Prior knowledge can be naturally incorporated in the choice of the nonlinear PI structure to improve the convergence rate.

- The mechanism of stabilization is the generation of attractive sets, instead of the more fragile requirement of convergence to specific equilibrium points.

- Our controller, being a simple nonlinear PI, has only a few parameters to tune; the role of these parameters on the (local) behavior of the system can usually be predicted - a property upon which hinges the practical success of fuzzy controllers.

The remaining of the paper is organized as follows. In Section 2 we present some practical examples that motivate our study, and give the precise definition of nonlinear PI used in the paper. The rationale of the design procedure is explained with a simple example in Section 3. Taking off from this example we compare in Section 4 the nonlinear PI approach with standard parameter adaptive control. The application of nonlinear PI to the problems with matched uncertainty and unknown control directions is presented in Sections 5 and 6 , respectively. We wrap up the paper with some concluding remarks and open problems.

Caveat emptor: Unless otherwise stated, the functions that appear throughout the paper are assumed to be sufficiently smooth to ensure global existence of solutions of the differential equations.

\section{Motivating examples}

In this section we present four motivating examples that have been studied in the literature, and for which our tools provide simple robust solutions.

\subsection{Eccentricity compensation}

In a recent interesting paper [4] the following eccentricity compensation problem was solved using an ingenious adaptive control technique.

Problem EC: Given the two-dimensional rotational system

$$
\ddot{y}=a \cos (b y+c)+u,
$$

where the angular position $y$ and velocity $\dot{y}$ are measurable, and $a, b, c$ are unknown positive parameters. Design a controller that ensures $\dot{y}(t)$ will asymptotically track any arbitrary bounded reference $\dot{y}_{*}(t)$ with known bounded first derivative. ${ }^{3}$

\subsection{Friction compensation}

Friction is an ubiquitous phenomenon in mechanical systems that is difficult to model and often requires to be compensated [23]. A basic (normalized) problem formulation, that covers the various models reported in [23], is as follows.

Problem FC: Given the two-dimensional mechanical system

$$
\ddot{y}=-F(\dot{y}, t)+u,
$$

\footnotetext{
${ }^{3}$ In fact, the result we present applies to the more general case where the system is described by the equation $\ddot{y}=\psi(y)+u$, with $\psi(\cdot)$ any bounded and (possibly) periodic function.
} 
where position $y$ and speed $\dot{y}$ are measurable, and the friction force $F$ is an unknown continuous function satisfying, for all $t \geqslant 0$, the bound

$$
|F(\dot{y}(t), t)| \leqslant M(1+|\dot{y}(t)|)
$$

with $M$ a known positive parameter. Design a controller that ensures $y(t)$ will asymptotically track any arbitrary bounded reference $y_{*}(t)$ with known bounded first- and second-order derivatives.

\subsection{Neural network function approximation}

Many papers in neural network control have been devoted to the solution of the following basic tracking problem.

Problem NN: Given the $n$-dimensional system

$$
\begin{aligned}
& \dot{x}_{1}=x_{2}, \\
& \dot{x}_{2}=x_{3}, \\
& \vdots \quad \vdots \\
& \dot{x}_{n}=\sum_{i=1}^{n} a_{i} x_{i}+\sum_{i=1}^{N} \frac{\delta_{i}}{1+\alpha_{i} \mathrm{e}^{-\beta_{i} x_{i}}}+u,
\end{aligned}
$$

where $N$ is a known positive integer, and all parameters $\left(a_{i}, i=1, \ldots, n\right),\left(\alpha_{i}>0, \beta_{i}>0, \delta_{i}>0, i=1, \ldots, N\right)$ are unknown. Design a state-feedback controller that ensures $x(t)$ will asymptotically track some desired bounded reference $x_{*}(t)$.

\subsection{Chain of integrators with unknown control direction}

Global stabilization of a chain of integrators with unknown sign of the high frequency gain using only partial state feedback is a basic control problem whose solution-using standard model reference adaptive control techniques-is extremely involved.

Problem CI: Given the $n$-dimensional linear time-invariant system

$$
\begin{aligned}
& \dot{x}_{1}=x_{2}, \\
& \dot{x}_{2}=x_{3}, \\
& \vdots \quad \vdots \\
& \dot{x}_{n}=b u,
\end{aligned}
$$

where $x_{1}$ and $x_{n}$ are measurable, and $b$ is an unknown parameter. Design a controller that drives $x(t)$ to zero for all initial conditions $x(0) \in \mathbb{R}^{n}$.

We will show in this paper that all these problems can be solved with nonlinear PI controllers, which are defined as follows.

Definition 1. Given a set of measurable signals $y \in \mathbb{R}^{n_{y}}$, references $y_{*} \in \mathbb{R}^{n_{y}}$, and a scalar manipulated variable $u \in \mathbb{R}$, define three mappings: 


$$
\begin{aligned}
& \beta_{\mathrm{P}}: \mathbb{R}^{n_{y}} \times \mathbb{R}^{n_{y}} \rightarrow \mathbb{R}^{q}, \\
& w_{\mathrm{I}}: \mathbb{R}^{n_{y}} \times \mathbb{R}^{n_{y}} \times \mathbb{R}^{q} \rightarrow \mathbb{R}^{q}, \\
& \beta: \mathbb{R}^{q} \times \mathbb{R}^{n_{y}} \times \mathbb{R}^{n_{y}} \rightarrow \mathbb{R},
\end{aligned}
$$

The triple $^{4}\left\{\beta_{\mathrm{P}}, w_{\mathrm{I}}, \beta\right\}$ defines a nonlinear PI controller via the $q$ th order dynamical system

$$
\begin{aligned}
& u=\beta\left(\beta_{\mathrm{P}}\left(y, y_{*}\right)+\beta_{\mathrm{I}}, y, y_{*}\right), \\
& \dot{\beta}_{\mathrm{I}}=w_{\mathrm{I}}\left(y, y_{*}, \beta_{\mathrm{I}}\right) .
\end{aligned}
$$

Remark 1. The classical linear PI scheme for single-input single-output systems is recovered from our definition setting $n_{y}=q=1$, and choosing the linear functions

$$
\begin{aligned}
& \beta_{\mathrm{P}}=k_{\mathrm{P}}\left(y-y_{*}\right), \\
& w_{\mathrm{I}}=k_{\mathrm{I}}\left(y-y_{*}\right), \\
& \beta=-\beta_{\mathrm{P}}-\beta_{\mathrm{I}},
\end{aligned}
$$

where the constants $k_{\mathrm{P}}$ and $k_{\mathrm{I}}$ are the proportional and integral tuning gains. Remark that, according to the above definition, it is possible to include a "leakage" factor in the integral action.

\section{Rationale of the controller design}

To illustrate the rationale of the proposed design procedure we will consider the problem of regulation to zero of the simplest scalar system

$$
\dot{y}=\phi(y)+u,
$$

where $\phi(y)$ is an unknown continuous function that ranges in the interval $0 \leqslant \phi(y)<1$. (A word of caution is in order: although the derivations below will seem to be contrived, and tailored to this particular problem, we will show in the next sections that the same philosophy applies, mutatis mutandis, to other cases-including all the motivating examples of Section 2.)

First, we select some desired dynamics that we would like to impose to our system, in this case we choose $\dot{y}=-\lambda y$, with $\lambda>0$. Defining the signal ${ }^{5}$

$$
z=\beta_{\mathrm{P}}(y)+\beta_{\mathrm{I}}
$$

and closing the loop with our nonlinear PI (5), we can write the system dynamics in perturbed form as

$$
\dot{y}=-\lambda y+[\phi(y)+\beta(z, y)+\lambda y] .
$$

\footnotetext{
${ }^{4}$ For ease of exposition we will omit the arguments of the functions whenever these are clear from the context.

${ }^{5}$ As discussed in [3], viewing nonlinear PI controllers from an immersion and invariance (I\& I) viewpoint, this signal describes the behavior of the off-the-manifold dynamics. In spite of its nice geometric context, and for the sake of brevity, we will not pursue the I\& I perspective here.
} 
Our control objective is then to drive the term in brackets asymptotically to zero. Towards this end, we pose the following:

"Root searching" problem: Find functions $\left\{\beta_{\mathrm{P}}, w_{\mathrm{I}}, \beta\right\}$ that ensure

(i) for each $y$, there exists (at least one) $\bar{z}_{y}$ solution of the algebraic equation ${ }^{6}$

$$
\phi(y)+\beta\left(\bar{z}_{y}, y\right)+\lambda y=0,
$$

(ii) $z(t)$ converges asymptotically towards $\bar{z}_{y}$.

Let us now study the $z$ dynamics, which is described by

$$
\dot{z}=w_{\mathrm{I}}(y)+\frac{\partial \beta_{\mathrm{P}}}{\partial y}[\phi(y)+\beta(z, y)] .
$$

In view of the requirement (ii) we would like to enforce the "root" of the disturbance term, $\bar{z}_{y}$, as an equilibrium of the $z$ dynamics. Towards this end, we fix the integral parameter of our nonlinear PI as

$$
w_{\mathrm{I}}=\frac{\partial \beta_{\mathrm{P}}}{\partial y} \lambda y
$$

which yields

$$
\dot{z}=\frac{\partial \beta_{\mathrm{P}}}{\partial y}[\phi(y)+\beta(z, y)+\lambda y]
$$

To complete the description of the nonlinear PI we must define the functions $\beta_{\mathrm{P}}(y)$ and $\beta(z, y)$ to try to enforce conditions (i) and (ii) to the trajectories of the closed-loop system (8) and (9). For, consider the $z$ dynamics (9) as a system, parameterized in $y$, of the form $\dot{z}=f_{y}(z)$, where

$$
f_{y}(z) \triangleq \frac{\partial \beta_{\mathrm{P}}}{\partial y}[\phi(y)+\beta(z, y)+\lambda y]
$$

We will prove now that the problem will be solved if we can ensure that, uniformly in $y$, there exists $M>0$ such that

$$
z f_{y}(z)<0
$$

for all $|z|>M .^{7}$ Indeed, since for all values of $y$, the function $f_{y}(z)$ changes sign as $z$ ranges in $(-\infty,+\infty)$, it has at least one finite zero crossing. The function $f_{y}(z)$ will thus behave as depicted in Fig. 1, where the particular form of the graph in the gray area will depend on $y$ and the uncertain function $\phi(y)$, but will not be relevant for our analysis. It is easy to show that a function $\beta(z, y)$ that ensures $(10)$ is given by ${ }^{8}$

$$
\beta=-\lambda y-\frac{1}{1+\mathrm{e}^{-z / y}} .
$$

For frozen $y$ the first and the last roots of $f_{y}(z)$-namely, $\bar{z}_{y m}$ and $\bar{z}_{y M}$, respectively-are stable equilibria of the one-dimensional $z$ dynamics $(9)$, hence $\left[\bar{z}_{y m}, \bar{z}_{y M}\right]$ is a globally attractive interval. Of course, this

\footnotetext{
${ }^{6}$ We use the sub-index $(\cdot)_{y}$ to underscore the fact that the root will, in general, depend on $y$.

${ }^{7}$ It is important to stress that (10) is required only for large $z$. As will become clear later, this feature will distinguish our nonlinear PI from standard relay-based controllers.

${ }^{8}$ This function is not defined for $y=0$. See Remark 3 for an alternative, globally defined, function.
} 


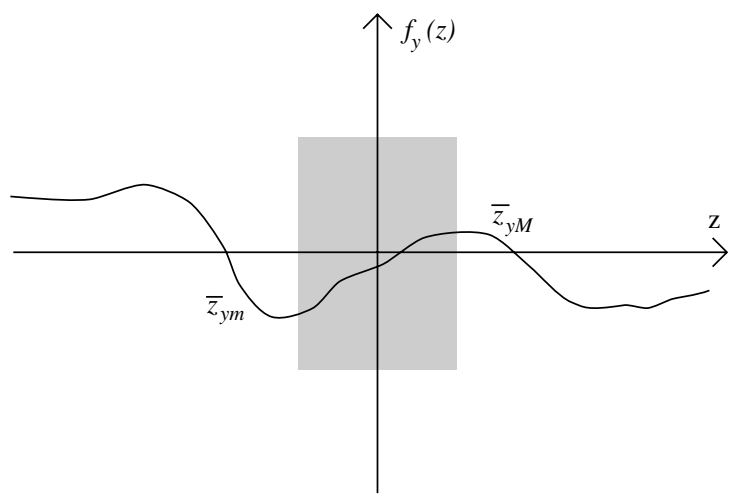

Fig. 1. The function $f_{y}(z)$.

simple reasoning cannot be extrapolated when $y$ changes, and a more careful analysis is needed to verify stability. As a first step, notice that

$$
\frac{\mathrm{d}}{\mathrm{d} t} z^{2}=2 z f_{y}(z)
$$

which, in view of (10), is negative for all $|z|>M$, hence $z$ is bounded-independently of the behavior of $y$. Now, consider the function $V(y, z)=\beta_{\mathrm{P}}(y)-z$, whose derivative is of the form ${ }^{9} \dot{V}=-\lambda\left(\partial \beta_{\mathrm{P}} / \partial y\right) y$. This simple calculation motivates the choice

$$
\beta_{\mathrm{P}}=\frac{1}{2} y^{2} .
$$

In this way, we have $V(y, z)=\frac{1}{2} y^{2}-z$, with $\dot{V}=-\lambda y^{2} \leqslant 0$. Since, we have shown above that $z$ is bounded, then $V(y, z)$ is bounded from below and we can conclude that $y$ is square integrable and bounded. To establish convergence of $y(t)$ to zero it suffices to prove that $y$ is uniformly continuous - for instance, showing that $\dot{y}$ is also bounded. Recalling that $\phi(y)$ is bounded (by assumption), this follows from (8) and the fact that $\beta(y, z)+\lambda y$ is also bounded.

We have thus established the following proposition.

Proposition 1. Consider the scalar system (6) with $\phi(y)$ such that, for all $y, 0 \leqslant \phi(y)<1$, in closed loop with the nonlinear PI controller (5) with the parameters

$$
\begin{aligned}
& \beta=-\lambda y-\frac{1}{1+\mathrm{e}^{-\left(\beta_{\mathrm{P}}+\beta_{\mathrm{I}}\right) / y}}, \\
& \beta_{\mathrm{P}}=\frac{1}{2} y^{2}, \\
& w_{\mathrm{I}}=\lambda y^{2} .
\end{aligned}
$$

Then, for all $\lambda>0$ and all initial conditions $\left(y(0), \beta_{\mathrm{I}}(0)\right), \lim _{t \rightarrow \infty} y(t)=0$ with all signals bounded.

\footnotetext{
${ }^{9}$ From (5) we see that $V=\beta_{\mathrm{I}}(y)$. Hence, the form of $\dot{V}$ is independent of the plant dynamics, and it stems only from our choice of $w_{\mathrm{I}}$, i.e. (7).
} 
Remark 2. The above nonlinear PI control law is discontinuous in $y$. If $\phi(0) \neq 0$, and without the knowledge of $\phi(0)$, it is clear that any controller that ensures stability for the whole class of admissible $\phi$ should be discontinuous. This obstruction appears in our construction because there does not exist function $\beta$, continuous in $y$, such that condition (10) holds. Indeed, as $y$ changes sign and $\phi$ remains with the same sign, the only way to enforce the inequality $z y[\phi(y)+\beta(z, y)+\lambda y]<0$ is that $\beta$ goes through a jump. (Selecting another function $\beta_{\mathrm{P}}$ will not help because $\partial \beta_{\mathrm{P}} / \partial y$ should have the same sign as $y$ to ensure $\dot{V} \leqslant 0$.) If $\phi(0)=0$ smooth stabilization is possible provided further information on $\phi$ is available, for instance, if a bound on its derivative at zero is known.

Remark 3. To implement the controller of Proposition 1 it is necessary to define the function $\beta$ as

$$
\beta(t)= \begin{cases}-\lambda y(t)-\left(1+\mathrm{e}^{\left.\frac{-\left(\beta_{\mathrm{P}}(t)+\beta_{\mathrm{I}}(t)\right)}{y(t)+\delta}\right)^{-1}}\right. & \text { if }\left\{y(t)>-\delta, \quad \exists t_{0}<t \text { s.t. } y\left(t_{0}\right) \leqslant-\delta,\right. \\ -\lambda y(t)-\left(1+\mathrm{e}^{\left.\frac{-\left(\beta_{\mathrm{P}}(t)+\beta_{\mathrm{I}}(t)\right)}{y(t)-\delta}\right)^{-1}}\right. & \text { and } y(\tau)<\delta, \quad \forall \tau \in\left[t_{0}, t\right], \\ & \text { if } y(t) \leqslant \delta, \quad \exists t_{0}<t \text { s.t. } y\left(t_{0}\right) \geqslant \delta \\ & \text { and } y(\tau)>-\delta \forall \tau \in\left[t_{0}, t\right],\end{cases}
$$

with $\delta$ a small positive number that defines the regulation error tolerance, e.g., determined by the noise level. This will generate a sliding regime in the set $\{(y, z) \mid y \in[-\delta, \delta], z \in[0, \infty)\}$, which turns out to be globally attractive. Convergence to this set, as usual in systems with discontinuous right-hand sides, happens in finite time.

Remark 4. Repeating the arguments of the proof of Proposition 1 it is easy to see that, with the same $\beta_{\mathrm{P}}(y)$ and $w_{\mathrm{I}}$, but scaling and shifting the activation function in $\beta$ as

$$
\beta=-\lambda y-\phi_{m}-\frac{\phi_{M}-\phi_{m}}{1+\mathrm{e}^{-\left(\beta_{\mathrm{P}}+\beta_{\mathrm{I}}\right) / y}},
$$

ensures global regulation for all $\phi_{m} \leqslant \phi(y) \leqslant \phi_{M}$.

Remark 5. It is clear from the proof that we have great freedom in the choice of the structure of the nonlinear PI. ${ }^{10}$ In particular, the only requirements on the function $\beta$ are that it is bounded and (10) holds, uniformly in $y$. Also, $\beta_{\mathrm{P}}$ can be any function such that $\partial \beta_{\mathrm{P}} / \partial y y \geqslant \delta y^{2}$, for some $\delta>0$. These degrees of freedom can be used to tailor the root searching in specific applications where additional information on $\phi(y)$ is available.

\section{Comparison with adaptive control}

To put our approach in perspective it is interesting to see how this simple example can be tackled with the classical parameter adaptive viewpoint. (We refer the reader to the lucid exposition of [19] for a unifying overview of the field, exhaustively covering the issues of parameterizations of linear systems and underscoring the importance of implicit tuning, a new class of tuning algorithms to which our nonlinear PI belongs.)

\footnotetext{
${ }^{10} \mathrm{We}$ have decided to illustrate the proposition with this activation function to make some connection with the neural networks literature.
} 
First, we assume a parameterization for the uncertainty, say $\phi(y)=\psi(y, \theta)$, where the function $\psi(y, \theta)$ is known, but the constant parameters $\theta \in \mathbb{R}^{r}$ are unknown. As pointed out in the Introduction, see also [10], to have a good function fit without a large number of parameters - that would stymie the convergence rate- the parameterization should be nonlinear. Then, we propose a certainty equivalent controller

$$
\begin{aligned}
& u=-\lambda y-\psi(y, \hat{\theta}), \\
& \dot{\xi}=f(y, \xi), \\
& \hat{\theta}=h(y, \xi),
\end{aligned}
$$

where $f(y, \xi), h(y, \xi)$ are continuous functions to be defined, and $\hat{\theta}$ plays the role of an estimate for $\theta$. In the indirect approach a bona fide parameter estimator that would, hopefully, drive $\hat{\theta}$ towards $\theta$, is built with some filters and approximation considerations for the calculation of a gradient descent (in the parameter space). On the other hand, in the direct approach we try to find instead a Lyapunov function candidate whose derivative we want to make nonpositive. The authors are not aware of an (smooth) adaptive solution to this problem, either direct or indirect, for the general class of functions $\phi(y)$ studied in Proposition 1. Simple solutions are, however, known for the (severely) restricted class of linearly parameterized functions, i.e., $\psi(y, \theta)=\psi_{0}(y) \theta$, with $\psi_{0}(y)$ known.

To pursue our comparison let us review the direct adaptive control solution, noting that similar conclusions can be drawn for indirect schemes. Consider the Lyapunov function candidate

$$
U(y, \hat{\theta})=\frac{1}{2}\left[y^{2}+\|\hat{\theta}-\theta\|^{2}\right],
$$

where $\|\cdot\|$ is the Euclidean norm. It is easy to see that picking $\dot{\hat{\theta}}=-\psi_{0}(y) y$ yields, $\dot{U}=-\lambda y^{2}$. The proof of global stability and convergence can be completed with some signal chasing, similar to the one used in the proof of Proposition 1 above.

The following remarks are in order.

- The direct adaptive control procedure hinges upon the exact cancellation of the uncertain terms in the Lyapunov function derivative. ${ }^{11}$ Leaving aside the fact that this operation is possible only under the critical assumption of linear parameterization, this is a very fragile operation which is the source of many robustness problems of adaptive schemes [16,9].

- In nonlinear PI a cascade connection between the plant dynamics (8) and the $z$ dynamics (9) is established. Although the latter plays a role similar to the term induced by the parameter error of adaptive control, in nonlinear PI we do not aim at its cancellation (or domination). Instead, we exploit the particular features of the nonlinear PI structure to drive it to zero.

- Following the classification of adaptive algorithms of [19], nonlinear PI is an identifier-based controller with implicit tuning. The former qualifier stems from the fact that $y \rightarrow 0$ without requiring $z \rightarrow 0$, while the implicit qualifier results from the simultaneous reduction of the tuning error, in this case $y$, and the design error, which is the disturbance term

$\phi(y)-\left[1+\mathrm{e}^{-\left(1 / 2 y^{2}+\beta_{1}\right) / y}\right]^{-1}$.

\section{Matched uncertainty}

In this section we extend the basic result of Section 3 of regulation of a first-order system with bounded uncertainty to tracking, high-dimensional systems (with unknown parameters) and unbounded uncertainty.

\footnotetext{
${ }^{11}$ Invoking high-gain arguments it is possible in some cases to dominate, instead of cancelling, this term.
} 
We also show how it is possible to address in a simple unified way the problems $2.1-2.3$ described in Section 2.

\subsection{Bounded uncertainty}

Proposition 2 (Tracking). Consider the first-order system (6) with $\phi(y)$ such that, for all $y, \phi_{m}<\phi(y)<\phi_{M}$, in closed loop with the nonlinear PI controller (5) with the parameters

$$
\begin{aligned}
& \beta=\dot{y}_{*}-\lambda \tilde{y}-\phi_{m}-\frac{\phi_{M}-\phi_{m}}{1+\mathrm{e}^{-\left(\beta_{\mathrm{P}}+\beta_{\mathrm{I}}\right) / \tilde{y}}}, \\
& \beta_{\mathrm{P}}=\frac{1}{2} \tilde{y}^{2}, \\
& w_{\mathrm{I}}=\lambda \tilde{y}^{2},
\end{aligned}
$$

with $\tilde{y} \triangleq y-y_{*}$ the output tracking error. Then, for all initial conditions $\left(y(0), \beta_{\mathrm{I}}(0)\right)$, and all bounded references $y_{*}(t)$, we have that $\lim _{t \rightarrow \infty} \tilde{y}(t)=0$, with all signals bounded.

Proof. Let $z=\beta_{\mathrm{I}}+\beta_{\mathrm{P}}$ and consider the dynamics ${ }^{12}$

$$
\begin{aligned}
& \dot{\tilde{y}}=-\lambda \tilde{y}+\left[\phi-\phi_{m}-\frac{\phi_{M}-\phi_{m}}{1+\mathrm{e}^{-z / \tilde{y}}}\right], \\
& \dot{z}=\tilde{y}\left[\phi-\phi_{m}-\frac{\phi_{M}-\phi_{m}}{1+\mathrm{e}^{-z / \tilde{y}}}\right] .
\end{aligned}
$$

Since condition (10) is satisfied we have, as before, that $z$ is bounded. Mimicking again the proof of Proposition 1 we consider $V(\tilde{y}, z)=\frac{1}{2} \tilde{y}^{2}-z$, with $\dot{V}=-\lambda \tilde{y}^{2}$. Given that $z$ is bounded, $V(\tilde{y}, z)$ is bounded from below and we can conclude that $\tilde{y}$ is square integrable and bounded. Convergence of $\tilde{y}(t)$ to zero follows immediately from boundedness of $\dot{\tilde{y}}$.

The extension of Proposition 1 to $n$-th order systems with unknown parameters and measurable state, studied for instance in $[1,22,8]$, is readily obtained as follows.

Proposition 3 (High-order systems with unknown parameters). Consider the n-dimensional single-input linear time-invariant system with matched uncertainty

$$
\dot{x}=A x+B[u+\phi(x)]
$$

with the following assumptions:

(i) There exists a (linear) output map $y=C^{\mathrm{T}} x$ such that $C^{\mathrm{T}} B \neq 0$ and we know $C^{\mathrm{T}} B$ and a bound on $\left|C^{\mathrm{T}} A\right|$. Moreover, $|y(t)|$ bounded implies $x(t)$ bounded.

(ii) The additive uncertainty $\phi(x)$ satisfies, uniformly in $x$, the bound $|\phi(x)| \leqslant \phi_{M}$.

\footnotetext{
${ }^{12}$ Notice that we have recovered again the structure of the motivation example, where the $\tilde{y}$ dynamics consists of a stable part plus a perturbation, and the $z$ dynamics has an equilibrium at the "roots" of the disturbance term. This situation will be repeated in all the cases considered in the paper.
} 
Then, the system in closed loop with a nonlinear PI controller with parameters

$$
\begin{aligned}
& \beta=\frac{1}{C^{\mathrm{T}} B}(1+\|x\|)\left(-y+M-\frac{2 M}{1+\mathrm{e}^{-\left(\beta_{\mathrm{P}}+\beta_{\mathrm{I}}\right) / y}}\right), \\
& \beta_{\mathrm{P}}=\frac{1}{2} y^{2}, \\
& w_{\mathrm{I}}=y^{2}(1+\|x\|),
\end{aligned}
$$

where $M \geqslant\left|C^{\mathrm{T}} A\right|+\left|C^{\mathrm{T}} B\right| \phi_{M}$, is such that, for all initial conditions $\left(x(0), \beta_{\mathrm{I}}(0)\right), \lim _{t \rightarrow \infty} x(t)=0$ with all signals bounded.

Proof. Let $z=\beta_{\mathrm{I}}+\beta_{\mathrm{P}}$ and write the closed-loop system as

$$
\begin{aligned}
& \dot{y}=-(1+\|x\|) y+(1+\|x\|)\left(M-\frac{2 M}{1+\mathrm{e}^{-z / y}}+\frac{C^{\mathrm{T}} A x+C^{\mathrm{T}} B \phi(x)}{1+\|x\|}\right), \\
& \dot{z}=y(1+\|x\|)\left(M-\frac{2 M}{1+\mathrm{e}^{-z / y}}+\frac{C^{\mathrm{T}} A x+C^{\mathrm{T}} B \phi(x)}{1+\|x\|}\right) .
\end{aligned}
$$

Given that

$$
0 \leqslant M+\frac{C^{\mathrm{T}} A x+C^{\mathrm{T}} B \phi(x)}{1+\|x\|} \leqslant 2 M
$$

and proceeding as in the proof of Proposition 1 , we have that $\mathrm{d}|z|^{2} / \mathrm{d} t \leqslant 0$ for sufficiently large $|z|$, hence $z$ is bounded.

Mimicking again the proof of Proposition 1 we consider $V(y, z)=\frac{1}{2} y^{2}-z$, with $\dot{V}=-\lambda(1+\|x\|) y^{2}$. Since, we have shown above that $z$ is bounded, then $V(y, z)$ is bounded from below and we can conclude that $y$ is square integrable and bounded. Given that $y$ is bounded, we also have that the state $x$ is square integrable and furthermore, in view of assumption (i), bounded. Convergence of $y(t), x(t)$ to zero follows immediately from boundedness of $\dot{y}, \dot{x}$, respectively.

Remark 6. The assumption $C^{\mathrm{T}} B \neq 0$ can be replaced by

$$
C^{\mathrm{T}} B=C^{\mathrm{T}} A B=C^{\mathrm{T}} A^{2} B=\cdots=C^{\mathrm{T}} A^{i-1} B=0,
$$

and $C^{\mathrm{T}} A^{i} B \neq 0$ and for some $i$ known, $C^{\mathrm{T}} x, C^{\mathrm{T}} A x, C^{\mathrm{T}} A^{(i-1)} x$ are measurable, and if we have a bound on $\left|C^{\mathrm{T}} A^{i}\right|$. In this case, we can repeat the proof of Proposition 3 taking instead of $y, C^{\mathrm{T}}\left(I+\alpha_{1} A+\cdots+\alpha_{i-1} A^{(i-1)}\right) x$, for some suitably chosen coefficients $\alpha_{i}$.

Remark 7. With some simple calculations it is possible to extend the result of Proposition 3 to force the output $y(t)$ to track signals generated by $\dot{x}_{*}=A_{*} x_{*}+B r, y_{*}=C_{*}^{\mathrm{T}} x_{*}$, for arbitrary known bounded $r(t)$.

\subsection{Unbounded uncertainty}

The technique developed in Section 3 can still be applied if the uncertainty is unbounded, but we know $M$ and $N$ such that

$$
|\phi(y)| \leqslant M\left(1+|y|+|y|^{N}\right)
$$

holds, uniformly in $y$. 
Proposition 4 (Unbounded uncertainty). Consider the scalar system (6), where $\phi(y)$ verifies (12), in closed loop with a nonlinear PI controller with parameters

$$
\begin{aligned}
& \beta=M\left(1+|y|+|y|^{N}\right)\left(-y+1-\frac{2}{1+\mathrm{e}^{-\left(\beta_{\mathrm{P}}+\beta_{\mathrm{I}}\right) / y}}\right), \\
& \beta_{\mathrm{P}}=\frac{1}{2} y^{2}, \\
& w_{\mathrm{I}}=-y^{2} M\left(1+|y|+|y|^{N}\right) .
\end{aligned}
$$

Then, for all initial conditions $\left(y(0), \beta_{\mathrm{I}}(0)\right), \lim _{t \rightarrow \infty} y(t)=0$ with all signals bounded.

Proof. The closed-loop dynamics is given by

$$
\begin{aligned}
& \dot{y}=-M\left(1+|y|+|y|^{N}\right) y+M\left(1+|y|+|y|^{N}\right)\left(1-\frac{2}{1+\mathrm{e}^{-z / y}}+\frac{\phi(y)}{M\left(1+|y|+|y|^{N}\right)}\right), \\
& \dot{z}=y M\left(1+|y|+|y|^{N}\right)\left(1-\frac{2}{1+\mathrm{e}^{-z / y}}+\frac{\phi(y)}{M\left(1+|y|+|y|^{N}\right)}\right),
\end{aligned}
$$

where we have used, again, the new coordinate $z=\beta_{\mathrm{I}}+\beta_{\mathrm{P}}(y)$, and the definitions of $\beta_{\mathrm{P}}$ and $w_{\mathrm{I}}$.

Given that condition (10) is satisfied and using the bound (12), the proof can be completed following verbatim the steps of the proof of the previous proposition.

Remark 8. Using the construction of Proposition 4 we can extend the results of Proposition 2 (tracking) and Proposition 3 (high order systems) to handle possibly unbounded functions $\phi$ that satisfy (12). These results are omitted for brevity.

\subsection{Examples}

We will illustrate now how the techniques developed above apply to the solution of some of the motivating problems of Section 2.

Problem EC: In this case the uncertain term is bounded, and the system is second order, hence Proposition 3 applies. Note however, that only velocity tracking is required, and this can be achieved by means of a nonlinear PI controller with parameters

$$
\begin{aligned}
& \beta=\ddot{y}_{*}-(1+\|x\|)\left(\dot{\tilde{y}}+M-\frac{2 M}{1+\mathrm{e}^{-\left(\beta_{\mathrm{P}}+\beta_{\mathrm{I}}\right) / y}}\right), \\
& \beta_{\mathrm{P}}=\dot{\tilde{y}}, \\
& w_{\mathrm{I}}=\dot{\tilde{y}}(1+\|x\|),
\end{aligned}
$$

where $\dot{\tilde{y}}=\dot{y}-\dot{y}_{*},\|x\|=\|(y, \dot{y})\|, M \geqslant a$, and $\dot{y}_{*}$ is the velocity reference to be tracked. Finally, it is clear that we do not to assume any particular form for the disturbance $\phi(\cdot)$, as the proposed controller will achieve asymptotic tracking for all bounded functions. This is in contrast with the result of [4], which requires the exact knowledge of the form (but not of the parameters) of the nonlinearity $\phi(\cdot)$.

Problem FC: This problem is conceptually similar to the Problem EC. The only difference is that the nonlinearity, representing the friction force, is linearly bounded, i.e., $|F(\dot{y}, t)| \leqslant M(1+|\dot{y}|)$. Hence, a combination of the results in Proposition 3 and Proposition 2 has to be used. The details are omitted for brevity. 
Problem NN: This problem can be treated as the problem FC, provided that the whole term

$$
\sum_{i=1}^{n} a_{i} x_{i}+\sum_{i=1}^{N} \frac{\delta_{i}}{1+\alpha_{i} \mathrm{e}^{-\beta_{i} x_{i}}}
$$

is regarded as an unknown perturbation. Note finally that, because of the simple structure of the system it is a trivial task to find an output map satisfying the hypothesis of Proposition 3.

\section{Unknown control directions}

The problem of stabilizing systems with unknown control directions, i.e., the high-frequency gain in the case of linear systems, has been extensively studied in the adaptive control literature, see [16,12] and the references therein. A major breakthrough in this problem was the introduction of the so-called Nussbaum gains, which similarly to our "root-searching" functions, continuously change sign until they latch to the right sign of the high-frequency gain. Although of significant theoretical interest, the poor robustness of this kind of schemes, elegantly revealed in [9], makes them practically inadmissible. In this section we show how our framework provides alternative, robust, solutions to this class of problems.

As before, we start with the simplest representative problem of stabilization of a scalar system, first for regulation and then for tracking. Finally, we present a partial state-feedback stabilizer for a chain of integrators.

\subsection{State feedback}

Proposition 5 (Regulation). Consider the scalar system

$$
\dot{y}=a y+b u,
$$

where $a$ and $b$ are unknown parameters, in closed loop with a nonlinear PI controller with parameters

$$
\begin{aligned}
& \beta=\left(\beta_{\mathrm{I}}+\beta_{\mathrm{P}}\right) \cos \left(\beta_{\mathrm{I}}+\beta_{\mathrm{P}}\right) y, \\
& \beta_{\mathrm{P}}=\frac{1}{2} y^{2}, \\
& w_{\mathrm{I}}=\lambda y^{2},
\end{aligned}
$$

with $\lambda>0$. Then, for all initial conditions $\left(y(0), \beta_{\mathrm{I}}(0)\right)$, we have that $\lim _{t \rightarrow \infty} y(t)=0$ with all signals bounded.

Proof. Replacing (14) in (13), and defining, as usual, $z=\beta_{\mathrm{I}}+\beta_{\mathrm{P}}$, we obtain the closed-loop dynamics

$$
\begin{aligned}
& \dot{y}=-\lambda y+[a+b z \cos (z)+\lambda] y, \\
& \dot{z}=y^{2}[a+b z \cos (z)+\lambda] .
\end{aligned}
$$

(We have added and subtracted $\lambda y$ in the first equation to underscore the perturbed dynamics structure.)

Notice that $\dot{z}=y^{2} f_{y}(z)$, with $f_{y}$ a function that has an infinite number of roots, which define bounded intervals that are invariant to the $z$ dynamics, ensuring that $z$ is bounded. ${ }^{13}$ See Fig. 2. The proof can be completed following verbatim the steps of the proof of the previous proposition considering the function $V(y, z)=\frac{1}{2} y^{2}-z$ whose derivative yields $\dot{V}=-\lambda y^{2}$ and chasing the signals to prove uniform continuity of $y$.

\footnotetext{
${ }^{13}$ This property holds true independently of the behavior of the "time-scaling" factor $y^{2}$.
} 


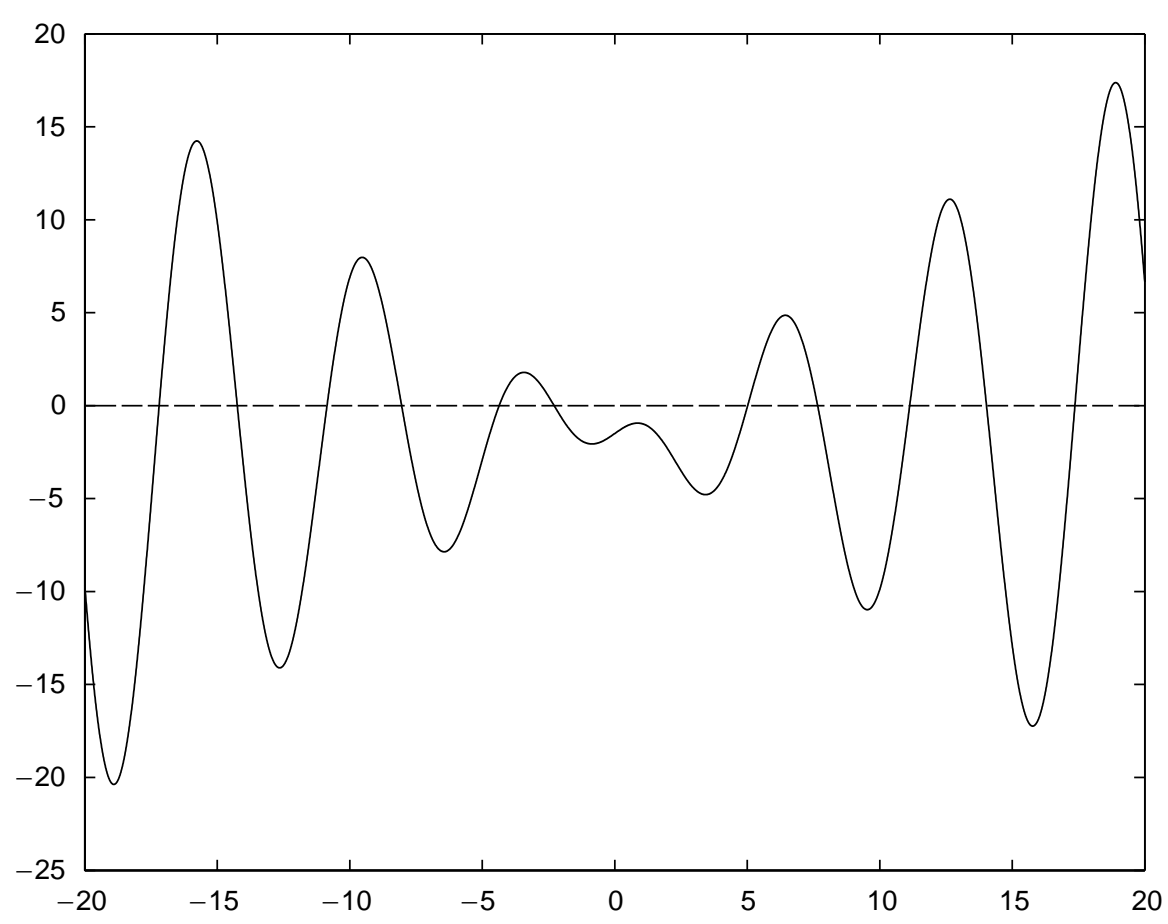

Fig. 2. Plot of the function $a+\lambda+b z \cos (z)$.

Proposition 6 (Tracking). Consider the scalar system $\dot{y}=b u$, with $b$ an unknown parameter, in closed loop with the nonlinear PI controller

$$
\begin{aligned}
& \beta=-\left(\beta_{\mathrm{I}}+\beta_{\mathrm{P}}\right) \cos \left(\beta_{\mathrm{I}}+\beta_{\mathrm{P}}\right)\left(\tilde{y}-\frac{1}{\lambda} \dot{y}_{*}\right), \\
& \beta_{\mathrm{P}}=\frac{1}{2} \tilde{y}^{2}-\frac{1}{\lambda} \dot{y}_{*} \tilde{y} \\
& w_{\mathrm{I}}=\frac{1}{\lambda} \ddot{y}_{*} \tilde{y}+\left(\tilde{y}-\frac{1}{\lambda} \dot{y}_{*}\right) \dot{y}_{*}+\lambda\left(\tilde{y}-\frac{1}{\lambda} \dot{y}_{*}\right)^{2},
\end{aligned}
$$

with $\lambda>0$. Then, for all initial conditions $\left(y(0), \beta_{\mathrm{I}}(0)\right)$, and all bounded references $y_{*}(t)$ with bounded firstand second-order derivative, we have that $\lim _{t \rightarrow \infty} \tilde{y}(t)=0$, with all signals bounded.

Proof. The closed-loop dynamics is now

$$
\begin{aligned}
& \dot{\tilde{y}}=-\lambda \tilde{y}+\left(\tilde{y}-\frac{1}{\lambda} \dot{y}_{*}\right)[-b z \cos (z)+\lambda], \\
& \dot{z}=\left(\tilde{y}-\frac{1}{\lambda} \dot{y}_{*}\right)^{2}[-b z \cos (z)+\lambda] .
\end{aligned}
$$

Arguing as in the proof of Proposition 5 we have that $z$ is bounded. However, the presence of the term $\frac{1}{\lambda} \dot{y}_{*}$ in the "time-scaling" factor of the $z$ dynamics, which cannot be removed because of the lack of knowledge 
of $b$, hampers the application of the usual Lyapunov function, and some additional arguments are needed to complete the proof.

Define $s=\tilde{y}-\frac{1}{\lambda} \dot{y}_{*}$, and write the $\tilde{y}$ dynamics in terms of $s$ as

$$
\dot{s}=-b z \cos (z) s-\dot{y}_{*}-\frac{1}{\lambda} \ddot{y}_{*} .
$$

Consider the function $W(s, z)=\frac{1}{2} s^{2}-z$, whose derivative yields

$$
\begin{aligned}
\dot{W} & =-\lambda s^{2}-s\left(\dot{y}_{*}+\frac{1}{\lambda} \ddot{y}_{*}\right) \\
& \leqslant-\frac{\lambda}{2} s^{2}+M,
\end{aligned}
$$

where $M \geqslant(1 / 2 \lambda)\left(\dot{y}_{*}+\frac{1}{\lambda} \ddot{y}_{*}\right)^{2}$. Now, from the boundedness of $z$, we have that there exists a positive constant $c_{1}$, such that $W \leqslant \frac{1}{2} s^{2}+c_{1}$. We then get the differential inequality $\dot{W} \leqslant-W+M+c_{1}$, from which we conclude that $W$, and consequently $s$ and $\tilde{y}$, are bounded.

We will now establish the convergence result. First, introducing the time scaling $\mathrm{d} \tau / \mathrm{d} t=s^{2}$, and looking at the $z$ dynamics in the $\tau$-time scale we see that the trajectories of (17) will converge to a constant $z_{\infty}$ such that $-b z_{\infty} \cos \left(z_{\infty}\right)+\lambda=0$ if and only if $\lim _{t \rightarrow \infty} \int_{0}^{t} s^{2}(\tau) \mathrm{d} \tau=\infty$. Hence, if $s$ is not square integrable we conclude from (16), and boundedness of $\tilde{y}, \dot{y}_{*}$, that $\tilde{y}(t) \rightarrow 0$. On the other hand, if $s$ is square integrable-and recalling that the term in square brackets in (16) is bounded-we can invoke Theorem 4.9 of [5] to conclude that $\tilde{y}(t)$, being the output of a strictly proper exponentially stable linear system with square integrable input, converges to zero. This completes the proof.

The following lemma allows to define a class of functions that could be used instead of $z \cos (z)$ in the PI function $\beta$ in Propositions 5 and 6 .

Lemma 1. Consider the scalar non-autonomous system $\dot{z}=\alpha(z)+m(t)$ with initial condition $z(0)=z_{0} \in \mathbb{R}$. Assume there exists two infinite sub-sequences $\mathscr{S}^{+} \triangleq\left\{z_{k}^{+}\right\} \rightarrow \infty$ and $\mathscr{S}^{-} \triangleq\left\{z_{k}^{-}\right\} \rightarrow-\infty$ such that, for all $k$,

$$
\begin{aligned}
& \sup _{t} m(t)+\alpha\left(z_{k}^{+}\right)<0, \\
& \inf _{t} m(t)+\alpha\left(z_{k}^{-}\right)>0 .
\end{aligned}
$$

Then, $z$ is bounded.

Proof. Given any initial condition $z_{0}$ there exists an element of $\mathscr{S}^{+}$, say $z_{j}^{+}$, such that $z_{j}^{+}>0$ and $z_{j}^{+}>z_{0}$. Since $m(t)+\alpha\left(z_{j}^{+}\right)<0$ for all $t$, we have that $z_{j}^{+}>z(t)$ for all $t>0$. A similar argument, using an element of the sequence $\mathscr{S}^{-}$, can be used to prove that $z(t)$ is also bounded from below, completing the proof.

Remark 9. The elegance and simplicity of Proposition 5 can hardly be overestimated. It should be contrasted with the complexity of the analysis of Nussbaum gain controllers [16] for the same problem. Moreover, the proposed controller will also stabilize plants of the form $\dot{y}=a y+\phi(y)+b u$, with $\phi(0)=0$ and bounded and differentiable $\phi$. Unfortunately, except for the case $a=0$, given in Proposition 6, we have not been able to extend this result to tracking. See [2] for an application of this construction to adaptive calibration for visual servoing. 


\subsection{Observer-based design}

For the sake of brevity we omit the presentation of the case of $n$-th order systems with full state feedback, and give directly the following output feedback result.

Proposition 7 (Partial state feedback). Given the n-dimensional stabilizable and detectable single-input singleoutput linear time-invariant system

$$
\begin{aligned}
& \dot{x}=A x+B b u, \\
& y=C^{\mathrm{T}} x,
\end{aligned}
$$

with the following assumptions:

(i) $A \in \mathbb{R}^{n \times n}, B, C \in \mathbb{R}^{n}$, and $b \in \mathbb{R}$ are unknown, but $B^{\mathrm{T}} B \in \mathbb{R}$ is known;

(ii) the only signals available for measurement are $y, B^{\mathrm{T}} x$ and $B^{\mathrm{T}} A x$;

(iii) there exist known matrix $F \in \mathbb{R}^{n \times n}$ and vectors $L, K \in \mathbb{R}^{n}$, such that the $2 n \times 2 n$ matrix

$$
\left[\begin{array}{cc}
A & B K^{\mathrm{T}} \\
L C^{\mathrm{T}} & F
\end{array}\right]
$$

is strictly Hurwitz. ${ }^{14}$

Under these conditions, an observer-based nonlinear PI controller with parameters

$$
\begin{aligned}
& \beta=\left(\beta_{\mathrm{I}}+\beta_{\mathrm{P}}\right) \cos \left(\beta_{\mathrm{I}}+\beta_{\mathrm{P}}\right) K^{\mathrm{T}} \xi, \\
& \beta_{\mathrm{P}}=x^{\mathrm{T}} B K^{\mathrm{T}} \xi, \\
& w_{\mathrm{I}}=-\xi^{\mathrm{T}} K B^{\mathrm{T}}\left(A x+B K^{\mathrm{T}} \xi\right)-x^{\mathrm{T}} B K^{\mathrm{T}}(F \xi+L y),
\end{aligned}
$$

and $\xi$ generated as

$$
\dot{\xi}=F \xi+L y,
$$

drives $x(t)$ to zero preserving all signals bounded for all initial conditions $\left(\beta_{\mathrm{I}}(0), \xi(0), x(0)\right.$.

Proof. Replacing (19) in the system dynamics we obtain the perturbed closed-loop system

$$
\left[\begin{array}{c}
\dot{x} \\
\dot{\xi}
\end{array}\right]=\left[\begin{array}{cc}
A & B K^{\mathrm{T}} \\
L C^{\mathrm{T}} & F
\end{array}\right]\left[\begin{array}{l}
x \\
\xi
\end{array}\right]+\left[\begin{array}{c}
B K^{\mathrm{T}} \xi[b z \cos (z)-1] \\
0
\end{array}\right] .
$$

Evaluating the time derivative of $z$, and using (19), we get

$$
\dot{z}=\xi^{\mathrm{T}} K B^{\mathrm{T}} B K^{\mathrm{T}} \xi[b z \cos (z)-1],
$$

from which we conclude that $z$ is bounded.

To complete the proof we proceed similarly to the convergence proof of Proposition 6. Namely, we consider first the case when $B K^{\mathrm{T}} \xi$ is not square integrable, which is necessary and sufficient for convergence to zero of the disturbance term $\varepsilon_{t} \triangleq b z \cos (z)-1$. Now, from (21), boundedness of $z$, and condition (iii), a simple Lyapunov argument allows to establish the existence of a matrix $P=P^{\mathrm{T}}>0$ such that the Lyapunov function

\footnotetext{
${ }^{14}$ This is tantamount to saying that the triple $\{A, B, C\}$ is dynamic output feedback stabilizable.
} 
$V(x, \xi)=\frac{1}{2} \operatorname{col}(x, \xi)^{\mathrm{T}} P \operatorname{col}(x, \xi)$ satisfies the bound

$$
\dot{V} \leqslant-\alpha\left(1-\varepsilon_{t}\right) V
$$

for some $\alpha>0$. Given that there is no finite escape time and $\varepsilon_{t} \rightarrow 0$, we conclude that $x(t)$ will converge to zero.

On the other hand, if $B K^{\mathrm{T}} \xi$ is square integrable, we also conclude $x(t) \rightarrow 0$ from (21), condition (iii), boundedness of $z$ and Theorem 4.9 of [5].

Remark 10. Proposition 7 directly provides the solution to Problem CI. Indeed, in the case of a chain of integrators we have

$$
A=\left[\begin{array}{cccc}
0 & 1 & \cdots & 0 \\
0 & 0 & \cdots & 0 \\
\vdots & \vdots & \cdots & \vdots \\
0 & 0 & \cdots & 1 \\
0 & 0 & \cdots & 0
\end{array}\right], \quad B=\left[\begin{array}{c}
0 \\
0 \\
\vdots \\
0 \\
1
\end{array}\right], \quad C=\left[\begin{array}{c}
1 \\
0 \\
\vdots \\
0 \\
0
\end{array}\right] .
$$

Hence, $B^{\mathrm{T}} x=x_{n}$ and $C^{\mathrm{T}} x=x_{1}$, while $B^{\mathrm{T}} A=0$. Finally, as $A$ and $B$ are fully known, assumption (iii) is trivially verified.

\subsection{Robustness}

It is interesting to compare the nonlinear PI derived in Proposition 5 with the well-known Nussbaum gain controller [16], which (in its basic form) is given by

$$
\begin{aligned}
& u=\hat{\theta}^{2} \cos (\hat{\theta}) y, \\
& \dot{\hat{\theta}}=\lambda y^{2} .
\end{aligned}
$$

Comparing with (5) and (14) we see that they differ on the presence of a proportional term $\beta_{\mathrm{P}}(y)$ in our controller, and the use of a quadratic term $\hat{\theta}^{2}$ in the Nussbaum scheme, instead of a linear term in the nonlinear PI. Although the latter difference is not essential, the proportional adaptation term effectively enhances the robustness of the nonlinear PI.

To illustrate this point let us consider the effect of unmodeled dynamics and place controller (5) and (14) in closed loop with an arbitrary $n$ th-order linear system $\dot{x}=A x+B u, y=C^{\mathrm{T}} x$. After some simple calculations we obtain the closed-loop dynamics

$$
\begin{aligned}
& \dot{x}=A x+B z \cos (z), \\
& \dot{z}=x^{\mathrm{T}} C^{\mathrm{T}}[\lambda x+A x+B z \cos (z)] .
\end{aligned}
$$

Of course, we still have the key property that the function $V(y, z)=\frac{1}{2} y^{2}-z$, has a derivative $\dot{V}=-\lambda y^{2} \leqslant 0$. Hence, as before, the central issue is when will $z$ remain bounded.

If we assume that the plant is relative degree one and minimum phase, then it admits a representation of the form

$$
\begin{aligned}
& \dot{\eta}=F \eta+G y, \\
& \dot{y}=H^{\mathrm{T}} \eta+a y+b u,
\end{aligned}
$$




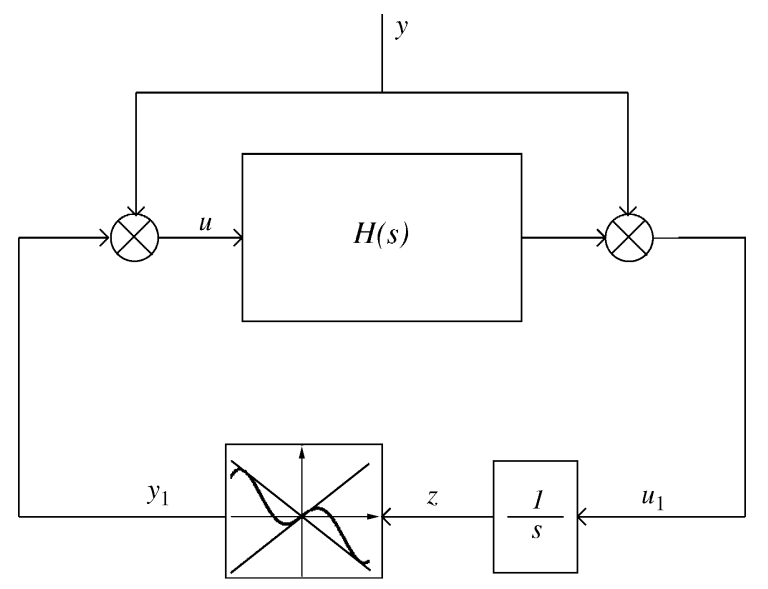

Fig. 3. Block diagram of system (23).

where $F$ is a strict Hurwitz matrix and $a, b, H$ and $G$ are arbitrary. The $z$ dynamics then becomes

$$
\dot{z}=y^{2}[a+b z \cos (z)+\lambda]+y H^{\mathrm{T}} \eta .
$$

Using the stability of $F$, and doing some simple bounding, we can prove that

$$
\dot{z} \leqslant \sup _{t \geqslant 0}|y(t)|^{2}[|a+b z \cos (z)+\lambda|+\kappa]+\varepsilon_{t},
$$

where $\kappa>0$ depends on the plant parameters, and $\varepsilon_{t}$ is an exponentially decaying term that depends on initial conditions. From the expression above we can conclude that $z$ is bounded, hence establishing the robustness of nonlinear PI vis-à-vis relative degree one minimum phase unmodeled dynamics.

The robustifying effect of the proportional term can also be highlighted adopting a passive systems viewpoint. For, let us represent system (23) by the block diagram of Fig. 3. It is easy to prove that, if $z$ is bounded, the feedback map $u_{1} \mapsto y_{1}$ described by

$$
\begin{aligned}
& \dot{z}=u_{1}, \\
& y_{1}=z \cos (z)
\end{aligned}
$$

is passive, with storage function $z \sin (z)+\cos (z)$. On the other hand, it is well known that the forward path is passive if the transfer function

$$
H(s)=C^{\mathrm{T}}\left[(\lambda I+A)(s I-A)^{-1}+I\right] B
$$

is positive real. Let us consider now the case when the plant is a simple integrator perturbed by a fast parasitic first-order unmodeled dynamics, that is, $Y(s)=(M / s(s+M)) U(s)$, where $M>0$. Via simulations and some analysis, it has been shown in [9] that the Nussbaum gain controller is unstable for $M>1$. In this case $H(s)=M s+\lambda / s(s+M)$, which is positive real for all $\lambda<M .{ }^{15}$ If we remove the proportional term, as in the Nussbaum gain controller, the zero disappears and we get $H(s)=\lambda M / s(s+M)$ which is clearly not positive real. This provides an alternative explanation of the poor robustness properties of Nussbaum gain controllers unveiled in [9].

\footnotetext{
${ }^{15}$ This fact by itself is not conclusive for stability-because passivity of the feedback operator relies on boundedness of $z$. However, using standard center manifold arguments, it is possible to show that the equilibria of closed-loop system, which are of the form $(0,0, \bar{z})$, are stable. Moreover, this equilibrium manifold is locally attractive.
} 
Before closing this section let us point out that it is well known that open integration of a quadratic signal may generate a parameter drift in the presence of (even zero mean) noise. Both schemes suffer from this clear drawback, however, the presence of the proportional term (instead of a quadratic) in the nonlinear PI makes it less sensitive to noise. Last, but not least, the stability analysis of Nussbaum gain controllers is far more complicated than the simple derivation given above, indicating that we have a better understanding of the stabilization mechanism of nonlinear PI.

\section{Conclusions}

We presented in this paper a radically new framework for stabilization of partially known systems. We considered, in particular, linear systems perturbed by coarsely known matched nonlinear uncertainties, a problem that has been typically approached in the adaptive control literature postulating a nonlinearly parameterized structure for the uncertainty, and implementing a parameter estimator that tries to match the data. Motivated by the difficulties associated to the problem of estimation of nonlinearly parameterized systems, we propose here to abandon the parameterization perspective, and generate instead a stable error equation with a perturbation function that exhibits at least one root. By suitably adjusting the proportional and integral gains of a nonlinear PI controller we, roughly speaking, make this root an attractive equilibrium. It is also shown that this approach applies as well to systems with unknown control directions, for which we generate a new class of controllers with proven robustness properties.

The approach proposed in this paper is relevant for the fundamental problem of output-feedback stabilization of linear time-invariant systems with reduced prior knowledge. As thoroughly studied in [16], besides the highly contrived model reference and pole placement adaptive control paradigms, there are two different methods to solve this problem: adaptive high-gain feedback, á la Willems and Byrnes [26,11], or dense (open-loop) searches in parameter space, as proposed by Martensson [18]. ${ }^{16}$ While the first approach is clearly restricted to minimum phase systems and suffers from high noise sensitivity, the convergence rate of the second method has shown to be extremely poor for practical applications. For these reasons there exists a widespread - though, not fully justified - belief that the only schemes of practical interest are parameter estimator-based.

In this respect, it is instructive to critically review some drawbacks of these schemes that are conspicuous by their absence in our nonlinear PI approach. The parameter update laws used in direct adaptive control are usually rationalized invoking a minimization problem that is recursively "solved" seeking along a gradient descent direction. As the plant dynamics are coupled with the identifier, good estimates of the gradient are possible only under very particular circumstances, giving rise to the highly restrictive positive real condition, that permeates all the basic research in robust adaptive control [16]. This ubiquitous assumption can be "swept under the rug" adding leakages and (dynamic) normalizations to the parameter update laws. While the former induces unpredictable complex behaviors to the closed loop [17], the latter forces the adaptation to slow down reducing the controller alertness. Since tracking (slowly time varying) uncertain parameters is the raison d'être of adaptive control, these fixes are of little practical interest. A similar scenario appears in the indirect approach, whose success hinges upon some kind of parameter convergence. The latter can only be achieved with persistency of excitation - unavailable, and even undesirable, in many applications — or switching off the adaptation gains, as it implicitly happens in least-squares algorithms.

We hope that the preliminary results reported here open up a new avenue of research for the control of unknown systems, placed between the two existing extremes to describe uncertainty: unstructured vs.

\footnotetext{
${ }^{16}$ A third, practically appealing, alternative is the supervisory control of Morse [20,21], which is however formulated under different assumptions.
} 
parameterized, which are adopted in robust and adaptive control, respectively. Such a middle-point alternative may lead to less conservative results for less contrived problems.

\section{References}

[1] A. Annaswamy, F. Skantze, A. Loh, Adaptive control of continuous-time systems with convex/concave parametrizations, Automatica 34 (1) (1998) 33-49.

[2] A. Astolfi, L. Hsu, M. Netto, R. Ortega, Two solutions to the adaptive visual servoing problem, IEEE Trans. Robotics Automat. (2002).

[3] A. Astolfi, R. Ortega, Immersion and invariance: a new tool for stabilization and adaptive control of nonlinear systems, Fifth IFAC Symposium on Nonlinear Control Systems, NOLCOS'01, St. Petersburg, Russia, July 4-6, 2001.

[4] C. Canudas de Wit, L. Praly, Adaptive eccentricity compensation, IEEE Trans. Control Systems Tech. 8 (5) (200) $757-766$.

[5] C. Desoer, M. Vidyasagar, Feedback Systems: Input-Output Properties, Academic Press, New York, 1975.

[6] V. Fomin, A. Fradkov, V. Yakubovich, Adaptive Control of Dynamical Systems, Nauka, Moscow, 1981 (in Russian).

[7] A. Fradkov, R. Ortega, G. Bastin, On semi-adaptive control of convexly parametrized systems, Internat. J. Adapt. Control Signal Process. 15 (4) (2001).

[8] M. French, Cs. Szepesvari, E. Rogers, Uncertainty, and model dependency in approximate adaptive nonlinear control, IEEE Trans. Automat. Control 45 (2) (2000) 353-358.

[9] T. Georgiou, M. Smith, Robustness analysis of nonlinear feedback systems: an input-output approach IEEE Trans. Automat. Control 42 (9) (1997) 1200-1221.

[10] N. Gershenfeld, The Nature of Mathematical Modeling, Cambridge University Press, Cambridge, 1999.

[11] A. Ilchmann, Non-Identifier-Based High-Gain Adaptive Control, Springer, London, 1993.

[12] A. Ilchmann, E.P. Ryan, Universal Lambda-tracking for nonlinearly perturbed systems in the presence of noise, Automatica 30 (1990) 337-346.

[13] Z.P. Jiang, et al., Robust nonlinear integral control by partial state and output feedback, Proceedings of the 39th IEEE Conference on Decision and Control, Sydney, Australia, December 2000, pp. 2084-2090.

[14] M. Krstic, I. Kanellakopoulos, P.K. Kokotovic, Nonlinear and Adaptive Control Design, Wiley, New York, 1995.

[15] A. Loh, A. Annaswamy, F. Skantze, Adaptation in the presence of a general nonlinear parametrization: an error model approach IEEE Trans. Automat. Control 44 (1999) 1634-1652.

[16] I. Mareels, J.W. Polderman, Adaptive Systems: An Introduction, Birkhauser, Berlin, 1996.

[17] I.M.Y. Mareels, S. Van Gils, J.W. Polderman, A. Ilchmann, Asymptotic dynamics in adaptive gain control, in: P.M. Frank (Ed.), Advances in Control, Highlights of ECC '99, Springer, London, 1999, pp. 29-63.

[18] B. Martensson, Adaptive stabilization, Ph.D. Thesis, Department of Automatic Control, Lund Institute of Technology, Sweden, 1986.

[19] A.S. Morse, Towards a unified theory of parameter adaptive control, IEEE Trans. Automat. Control 37 (1) (1992) 15-29.

[20] A.S. Morse, Supervisory control of families of linear set-point controllers-Part 1: exact matching IEEE Trans. Automat. Control $41(10)(1996)$

[21] A.S. Morse, Supervisory control of families of linear set-point controllers-Part 2: robustness IEEE Trans. Automat. Control 42 (11) (1997).

[22] M. Netto, A. Annaswamy, R. Ortega, P. Moya, Adaptive control of a class of nonlinearly parametrized systems using convexification, Internat. J. Control 73 (14) (2000) 1312-1321.

[23] H. Olsson, K.J. Astrom, C. Canudas de Wit, M. Gafvert, P. Lischinky, Friction models and friction compensation, European J. Control 4 (3) (1998) 176-195.

[24] R. Ortega, Some remarks on adaptive neuro-fuzzy systems, Internat. J. Adapt. Control Signal Process. 10 (1996) $79-83$.

[25] E. Skafidas, A. Fradkov, R. Evans, I. Mareels, Approximation based adaptive control for nonlinear systems under matching conditions, Automatica 34 (3) (1998) 287-299.

[26] J. Willems, C. Byrnes, Global adaptive stabilization in the absence of information on the sign of the high frequency gain, in: A. Benssousan, J.L. Lions (Eds.), Proceedings of the Sixth International Conference Analysis and Optimization of Systems, Springer, Berlin, 1984, pp. 49-57. 